\title{
Institutional review board perspectives on obligations to disclose genetic incidental findings to research participants
}

\author{
Catherine Gliwa, BA ${ }^{1,2}$, Ilana R. Yurkiewicz, MD³, Lisa Soleymani Lehmann, MD ${ }^{4-6}$, \\ Sara Chandros Hull, PhD ${ }^{2,7}$, Nathan Jones, PhD $^{8}$ and Benjamin E. Berkman, JD, MPH ${ }^{2,7}$
}

Purpose: Researchers' obligations to disclose genetic incidental findings (GIFs) have been widely debated, but there has been little empirical study of the engagement of institutional review boards (IRBs) with this issue.

Methods: This article presents data from the first extensive $(n=796)$ national survey of IRB professionals' understanding of, experience with, and beliefs surrounding GIFs.

Results: Most respondents had dealt with questions about GIFs (74\%), but only a minority (47\%) felt prepared to address them. Although a majority believed that there is an obligation to disclose GIFs (78\%), there is still not consensus about the supporting ethical principles. Respondents generally did not endorse the idea that researchers' additional time and effort (7\%), and lack of resources
(29\%), were valid reasons for diminishing a putative obligation. Most (96\%) supported a right not to know, but this view became less pronounced (63\%) when framed in terms of specific case studies.

Conclusions: IRBs are actively engaged with GIFs but have not yet reached consensus. Respondents were uncomfortable with arguments that could be used to limit an obligation to return GIFs. This could indicate that IRBs are providing some of the impetus for the trend toward returning GIFs, although questions remain about the relative contribution of other stakeholders.

Genet Med advance online publication 19 November 2015

Key Words: genomic sequencing; incidental findings; institutional review board; research ethics

\section{INTRODUCTION}

Genetic sequencing technologies have become an increasingly powerful and affordable research tool, ${ }^{1}$ but large-scale wholegenome and whole-exome studies raise questions about how to manage genetic incidental findings (GIFs). ${ }^{2}$ GIFs are defined here as individual genetic results that are generated in the course of research but are unrelated to the aims of the research. ${ }^{3}$ There has been an active debate about the circumstances, if any, under which there is an obligation for researchers to disclose GIFs to research participants. ${ }^{4-7}$ Despite widespread recognition of the importance of this question, decisions about disclosure of GIFs are typically determined at an institutional level, often by institutional review boards (IRBs). There are currently no nationally accepted guidelines to direct researchers' or IRB professionals' decisions about disclosure of GIFs discovered during research. ${ }^{8}$

This study represents the first extensive national examination of IRB professionals' understanding of, experience with, and beliefs surrounding GIFs in the context of genomic sequencing. Prior studies have focused primarily on results generated from genome-wide association studies, ${ }^{9}$ which are less likely to produce GIFs when compared with whole-genome and whole-exome sequencing, ${ }^{10}$ or have used qualitative methodologies, interviewing limited numbers of subjects. ${ }^{11}$ Our goal was to capture-quantitatively and on a broader scale-how the research ethics community is currently thinking about the management and disclosure of GIFs in the context of genomic sequencing protocols. Specifically, we explored IRB professionals' perspectives on whether there is an obligation to return individual genetic results, the ethical principles that the research ethics community appeals to in support of such an obligation, and the reasons that might diminish any potential obligations. We also explored how views on incidental findings vary based on demographic characteristics such as professional training, IRB role, and genetic literacy.

\section{MATERIALS AND METHODS}

\section{Study participants and survey distribution}

We conducted a cross-sectional online survey of individuals sampled from the membership of Public Responsibility in Medicine and Research, a national nonprofit organization comprising individuals and organizations involved in various kinds of human subjects research. Its membership includes IRB members and other IRB professionals such as administrators and consultants, in addition to researchers and government staff.

The first two authors are co-first authors.

${ }^{1}$ David Geffen School of Medicine at UCLA, Los Angeles, California, USA; ${ }^{2}$ Department of Bioethics, Clinical Center, National Institutes of Health, Bethesda, Maryland, USA; ${ }^{3}$ Harvard Medical School, Boston, Massachusetts, USA; ${ }^{4}$ Department of Medicine, Brigham and Women's Hospital, Boston, Massachusetts, USA; ${ }^{5}$ Department of Global Health and Social Medicine, Harvard Medical School, Boston, Massachusetts, USA; ${ }^{6}$ Department of Health Policy and Management, Harvard T.H. Chan School of Public Health, Boston, Massachusetts, USA; ${ }^{7}$ Bioethics Core, National Human Genome Research Institute, National Institutes of Health, Bethesda, Maryland, USA; ${ }^{8}$ University of Wisconsin Survey Center, Madison, Wisconsin, USA. Correspondence: Benjamin E. Berkman (berkmanbe@mail.nih.gov) 
The survey was piloted using a small cohort of research ethics scholars prior to its wide distribution. A link to a self-administered electronic survey consisting primarily of multiple-choice questions, with some opportunity for short open-ended text answers, was emailed to 2,288 members of Public Responsibility in Medicine and Research who had previously self-identified as having a specific interest in human subject protections. Participants were provided with a $\$ 5$ preincentive. The response rate was calculated in accordance with RR2 from the American Association for Public Opinion Research ${ }^{12}$; therefore, only partial and complete surveys counted as responses. With $796 \mathrm{com}$ pleted surveys received, the response rate (RR2) was $34.8 \%$.

\section{Survey instrument}

Participants were introduced to the subject through a brief synopsis about the possibility of GIFs being generated from wholegenome and whole-exome sequencing. Participants also were provided with definitions of key terms used throughout the survey. The survey consisted of questions addressing seven central domains: (i) background and experience with GIFs, (ii) reasons that support an obligation to disclose GIFs, (iii) reasons that diminish an obligation to disclose GIFs, (iv) kinds of GIFs that should be disclosed, (v) informed consent and procedures for disclosing GIFs, (vi) study design and "loopholes," and (vii) the future of genomic research (Supplementary Appendix online).

Respondents who expressed a belief that researchers have an obligation to disclose GIFs to research participants were asked about reasons supporting that obligation. All respondents, regardless of how they answered that initial question, were asked about reasons that diminish an obligation to return GIFs. Participants indicated the strength of their agreement with various statements on a five-point Likert scale. Respondents were also given an "other" option, with space to write additional reasons. Participants were then presented with hypothetical scenarios involving various types of GIFs and study protocols, and were asked to indicate whether there was an obligation to disclose the GIF and whether the various studies should be approved.

Finally, the survey assessed demographic characteristics, background and experience with GIFs, and beliefs about additional guidance. Respondents also completed a 10-item genetic literacy scale adapted from Kaphingst et al. ${ }^{13}$ that tested knowledge in two domains (sequencing limitations and sequencing benefits).

\section{Data analysis}

Survey data were exported from Qualtrics, and survey responses were analyzed with descriptive statistics (percentages for discrete responses, means and standard deviations for continuous responses). Summary statistics were calculated using Stata 13 (StataCorp, College Station, TX). Differences among groups' responses were calculated by IRB voting status; genetic literacy score; educational level; primary role on the IRB (clinical, scientific, and all others); and combined primary and secondary IRB roles (clinical primary or secondary role, scientific but not clinical, and not clinical or scientific). Confidence intervals for the proportions were calculated and compared using a two-tailed $t$-test for differences. Differences with $P<0.05$ were considered statistically significant. The genomic literacy score was calculated for each domain (limits and benefits). Correct answers where the respondent was confident ("definitely") scored two points, and less confident correct answers ("probably") scored one point. The cumulative scores were grouped into low (0-5), moderate (6-9), and high (10) literacy groups.

\section{RESULTS}

\section{Respondent characteristics}

The majority of respondents identified as female (74\%) and non-Hispanic white (88\%). These characteristics are largely consistent with those found in survey research on similar IRBaffiliated populations ${ }^{14}$ (Table 1 ). The subject pool was generally well educated, with most respondents (67\%) holding a master's or doctoral degree. Most respondents (99\%) had been involved with an IRB for at least 1 year, and 36\% had been involved for more than 10 years. Of respondents who identified their current role or most recent affiliation with an IRB, 492 (68\%) held a nonvoting position, and 235 (32\%) served as a voting member of the IRB. Of voting members, 111 (15\%) reported filling a chair or vice-chair role. Other voting members reported being

\section{Table 1 Demographic characteristics}

\begin{tabular}{lc}
\hline Gender & \\
Female & $576(74 \%)$ \\
Male & $202(26 \%)$ \\
\hline $\begin{array}{l}\text { Education } \\
\text { High school or less }\end{array}$ \\
Some college & $3(1 \%)$ \\
College & $51(6 \%)$ \\
Master's degree & $208(26 \%)$ \\
Doctorate & $284(35 \%)$ \\
Race & $248(31 \%)$ \\
Caucasian & \\
African American & $673(88 \%)$ \\
Asian American & $46(6 \%)$ \\
American Indian & $27(4 \%)$ \\
Time with IRB (years) & $14(2 \%)$ \\
\hline 1 & \\
1-2 & $10(1 \%)$ \\
$3-5$ & $68(9 \%)$ \\
$6-10$ & $154(21 \%)$ \\
$\geq 10$ & $233(32 \%)$ \\
Role with IRB & $263(36 \%)$ \\
Chair or vice chair & \\
Scientific member & $110(15 \%)$ \\
Nonscientific member & $60(8 \%)$ \\
Administrator & $34(5 \%)$ \\
Other & $13(2 \%)$ \\
\hline
\end{tabular}

IRB, institutional review board. 
scientific members (9\%), nonscientific members (5\%), community members (2\%) and unspecified members (1\%). Nonvoting affiliates were primarily staff/administrators (60\%); the rest (8\%) filled other roles such as quality assurance and regulatory compliance.

The majority of our participants (74\%) reported having experience dealing with GIFs, suggesting that their survey responses were informed by experience (Table 2). Respondents expressed moderate confidence in their understanding of ethical issues raised by GIFs (19\% were very confident, $52 \%$ were somewhat confident, $35 \%$ were slightly confident) and slightly lower confidence in their genomic knowledge (9\% were very confident, $36 \%$ were somewhat confident, $35 \%$ were slightly confident). This self-assessment was in line with genetic literacy scores. ${ }^{13}$ When asked about limits of genomic sequencing, only $18 \%$ of respondents fell into the high literacy category (56\% had medium literacy, $26 \%$ had low literacy). Slightly more than half of the respondents (51\%) had high literacy regarding the benefits of genomic sequencing (36\% had medium literacy, $12 \%$ had low literacy). Most subjects (73\%) had received some training about GIFs, though among those $37 \%$ described having only

Table 2 Experience with genetic incidental findings

\begin{tabular}{lc}
\hline Has experience with GIFs & $532(74 \%)$ \\
Genomic knowledge & $74(9 \%)$ \\
Very confident & $288(36 \%)$ \\
Somewhat confident & $276(35 \%)$ \\
Slightly confident & $156(20 \%)$ \\
Not at all confident & \\
Ethical knowledge & $154(19 \%)$ \\
Very confident & $416(52 \%)$ \\
Somewhat confident & $177(22 \%)$ \\
Slightly confident & $47(6 \%)$ \\
Not at all confident & \\
\hline Training for evaluating questions about GIFs & $43(5 \%)$ \\
A lot & $252(32 \%)$ \\
Some & $285(36 \%)$ \\
A little & $213(27 \%)$ \\
None & \\
Preparedness for evaluating questions about GIFs & $64(8 \%)$ \\
Very well & $308(39 \%)$ \\
Somewhat well & $247(31 \%)$ \\
Slightly well & $173(22 \%)$ \\
Not at all & \\
Genomic literacy (limitations) & $208(26 \%)$ \\
Low & $442(56 \%)$ \\
Moderate & $140(18 \%)$ \\
Lenomic literacy (benefits) & $409(52 \%)$ \\
Moderate & $286(36 \%)$ \\
\hline
\end{tabular}

GIF, genetic incidental finding. “a little" training. About half (47\%) felt at least somewhat well prepared to grapple with GIFs.

\section{A duty to disclose GIFs}

In general, respondents indicated that researchers have some obligation to disclose GIFs to participants. The majority (65\%) indicated that there was "sometimes" an obligation to disclose GIFs; $13 \%$ indicated that there was "always" an obligation, and another 13\% indicated that there was "rarely" an obligation. Only $2 \%$ believed there was never an obligation, and $7 \%$ did not know.

Respondents who were grouped into the high genomic literacy cohort (limits scale only) were slightly more likely to be guarded in their agreement of an obligation to disclose GIFs. Although the total percentage of participants who stated that there was always or sometimes an obligation was generally consistent across genomic literacy levels (low $=82 \%$, medium $=$ $80 \%$, high $=72 \%$ ), the strength of agreement varied by genomic literacy. Participants in the high literacy group were less likely $(5 \%)$ to answer "yes, always" than those in the low literacy groups $(29 \% ; P>0.05)$.

\section{Ethical reasoning}

Respondents were divided when asked about the ethical principles that might support an obligation to disclose GIFs (Table 3). The principle with the strongest support was a duty to warn, with $84 \%$ of respondents either strongly agreeing or agreeing that researchers should disclose GIFs because of a duty to warn participants if they are in significant, imminent danger. Other principles that were widely supported included respect for autonomy of participants ( $80 \%$ of respondents strongly agreed or agreed) and beneficence (79\% strongly agreed or agreed). As a cross-check, the participants were asked to rank the top three principles that supported an obligation to disclose GIFs. The top-cited principles remained beneficence, respect for autonomy, and duty to warn. Respondents were more divided in their agreement versus disagreement on other principles, including professional responsibility (67 vs. $23 \%$ ), a need to maintain public trust in research (58 vs. $32 \%$ ), a need to maintain an institution's professional reputation (36 vs. $52 \%$ ), a need

Table 3 Ethical principles in support of an obligation to disclose

\begin{tabular}{lc} 
& Strongly agree or agree \\
\hline Duty to warn & $658(84 \%)$ \\
\hline Respect for autonomy & $626(80 \%)$ \\
\hline Beneficence & $615(79 \%)$ \\
\hline Professional responsibility & $520(67 \%)$ \\
\hline Public trust in research & $450(58 \%)$ \\
\hline Right to know & $418(54 \%)$ \\
Institutional reputation & $278(36 \%)$ \\
\hline Legal liability & $267(34 \%)$ \\
Participants = patients & $264(34 \%)$ \\
\hline Reciprocity & $261(34 \%)$ \\
\hline
\end{tabular}


to treat research participants like clinical patients (34 vs. 54\%), and reciprocity ( 34 vs. 56\%). Even though professional responsibility and public trust in research garnered more than $50 \%$ agreement, close examination of the top three ranking reasons reveals that support for these principles is lower than support for duty to warn, beneficence, and autonomy based on the relative percentages of first- and second-place rankings (Figure 1).

A number of participants indicated that they were unsure about certain principles. Most significantly, $18 \%$ were unsure about whether a concern for legal liability supported an obligation to disclose, and $13 \%$ were unsure about whether participants' right to know their own genetic information supported an obligation to disclose. There was some variability related to genomic literacy, with respondents with low literacy being significantly more likely $(P<0.05)$ to endorse certain principles (participants should be treated like patients, professional responsibility, and duty to warn) than respondents with high literacy.

There were only two potential factors that respondents strongly endorsed as diminishing an obligation to disclose GIFs (Table 4). Respondents were significantly more likely ( $P$ $<0.05$ ) to endorse inadequate clinical and analytical validity of the genetic screening information $(71 \%)$ and inadequate demonstrated clinical utility of the genetic risk information (66\%)

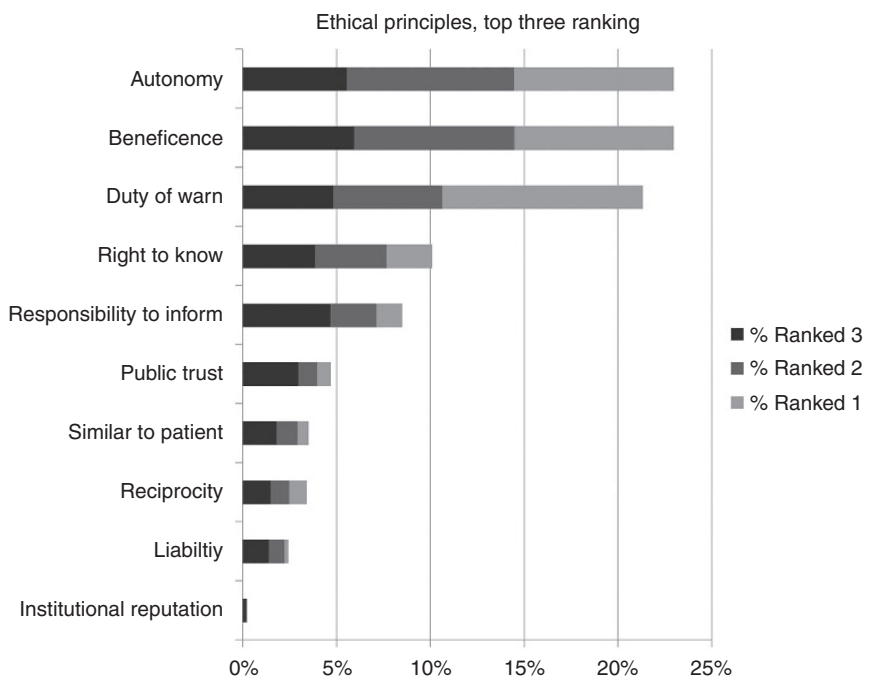

Figure 1 Ranking of the top three ethical principles.

Table 4 Factors that can diminish an obligation to disclose genetic incidental findings

\begin{tabular}{lc}
\hline Inadequate clinical or analytic validity & $\begin{array}{c}\text { Strongly agree } \\
\text { or agree }\end{array}$ \\
\hline Inadequately demonstrated clinical utility & $567(71 \%)$ \\
Lack of funding, resources, or infrastructure & $523(66 \%)$ \\
Participants won't understand & $230(29 \%)$ \\
Adverse psychological impact & $185(23 \%)$ \\
Researchers $\neq$ clinicians & $171(22 \%)$ \\
\hline Time and effort required & $145(18 \%)$ \\
\hline
\end{tabular}

as valid reasons for reducing an obligation. For the other factors considered, respondents did not believe they negatively affected an obligation to disclose GIFs. Most significantly, only $7 \%$ of respondents either agreed or strongly agreed that the additional time and effort required for the researcher to disclose GIFs was great enough to reduce an obligation to do so. In addition, only $18 \%$ of respondents agreed or strongly agreed with the idea that clinical researchers have different responsibilities from those of practicing physicians and thus do not have an obligation to disclose GIFs. Respondents generally also did not accept (23\% agreed or strongly agreed) that participants are not likely to sufficiently understand genetic risk information to disclose GIFs, and they did not indicate (22\% agreed or strongly agreed) that the potential psychological impact on participants of learning their genetic risk information is too high. Finally, only $29 \%$ of respondents agreed or strongly agreed that a lack of funding, resources, or infrastructure can diminish an obligation to disclose GIFs. There was some variability related to genomic literacy; respondents with high literacy were significantly more likely $(P<0.05)$ than respondents with low literacy to identify certain reasons that reduce an obligation to disclose (inadequate scientific validity, inadequate clinical validity, and scientists having different responsibilities than clinicians).

\section{Right not to know}

Almost all respondents (96\%) indicated that it is either definitely or probably acceptable for a participant to elect not to receive any GIFs. These views shifted, however, when applied to specific case studies. Case A involved a participant who had chosen not to receive any GIFs, but whose research team had identified genetic evidence of hereditary nonpolyposis colon cancer (a serious and actionable disease, also known as Lynch syndrome). In this case, $26 \%$ of respondents replied that the researchers should definitely or probably disclose this finding, $63 \%$ replied that researchers should definitely not or probably not disclose this finding, and $11 \%$ were unsure. Case B was similar to case A, but the original participant was deceased, and researchers were debating whether to inform the deceased participant's family about this potentially significant information. In this case, $51 \%$ of respondents believed that researchers should definitely or probably contact the family, $35 \%$ thought that researchers should definitely not or probably not contact the family, and $14 \%$ were unsure.

\section{DISCUSSION}

Questions about whether researchers have a responsibility to disclose GIFs have been widely debated in the literature. This is the first national survey in the United States to empirically examine IRB professionals' empirical understanding of GIFs and beliefs about researchers' obligations to disclose GIFs. Our data demonstrate that a majority of IRB professionals believe that researchers do have a duty to disclose GIFs to research participants. Underlying this perspective is a broad range of opinions about the ethical underpinnings of a duty to disclose 
GIFs and the various considerations that may or may not limit such a duty.

When the research ethics community began thinking about incidental findings, genomic sequencing technology was in its infancy. The turnaround time from obtaining blood to generating results was long, the cost was prohibitive, and the meaning of much of the information was uncertain. There were real questions about the frequency with which researchers would generate results that would be meaningful to individual participants. Today, with several of these limitations overcome, incidental findings have become more common, and the line between research and clinical care has continued to blur. Unsurprisingly, given recent advances in sequencing power, our data suggest that most IRBs today are actively dealing with GIFs; threequarters of respondents indicated some experience in thinking through these issues. This is no longer a theoretical problem, and we assume that many of our participants' responses were informed by their actual experiences.

It seems, however, that IRBs are only moderately prepared to face the problems posed by incidental findings. Although $73 \%$ of respondents had received some training about GIFs, about 36\% described this as "a little" training. Moreover, while a majority $(71 \%)$ were very or somewhat confident about their knowledge of the ethical issues GIFs raise, many respondents seemed to desire additional information about genomic science. Only $47 \%$ were very or somewhat confident about their genomic knowledge. Overall, respondents reported that they were only moderately prepared to deal with issues raised by GIFs, with fewer than half (47\%) indicating that they were very well prepared or somewhat well prepared.

\section{To what extent do IRBs think that there is a duty to disclose GIFs?}

Our survey began with a threshold question about whether there is a general duty to offer to disclose incidental findings to participants. Although a majority (78\%) of study respondents agreed that there was either sometimes or always an obligation to disclose GIFs, our data indicate that there is still not complete agreement on this issue. Importantly, a minority of respondents (15\%) indicated that there is either rarely or never an obligation to disclose GIFs. This may reflect a general uncertainty or apprehension about the state of genomic science and clinical genomics-a supposition supported by the majority of participants who agreed that a mistrust of scientific accuracy and clinical utility were factors that may reduce an obligation to disclose GIFs. This could also represent a manifestation of the spectrum of approaches to disclosure of GIFs, from researchfocused to autonomy-focused, as described by Ravitsky and Wilfond. ${ }^{15}$ Whatever the reason for this division, our data reflect a split similar to that expressed in the literature, providing a good sense for the distribution of IRB support for and against an obligation to offer incidental findings to participants. Although we hypothesized that views on the existence of an obligation to disclose incidental findings would vary by professional training (e.g., MD versus $\mathrm{PhD}$ ) and role (e.g., clinical versus scientific), there were no statistically significant differences between these groups. There was a modest relationship between genetic literacy (limits scale) and the strength of a perceived duty to disclose; respondents with high literacy were less likely than those with low literacy to indicate that there is always an obligation to return genetic findings.

\section{What ethical reasons are cited in support of an obligation to disclose GIFs?}

Consensus about the scope of researchers' obligations regarding incidental findings has been elusive. This lack of agreement might be due to the fact that the contours of an obligation necessarily shift depending on the underlying principle(s) one puts forward. ${ }^{16,17}$ To learn more about how IRBs are thinking through the problem of incidental findings, we asked survey participants about the principles they endorse in support of an obligation to disclose GIFs. While IRB members' views on certain principles are not dispositive proof of the correct normative view, such data can provide an insight into their ethical reasoning.

Respondents did not endorse a single dominant principle in support of an obligation to disclose GIFs. The three ethical principles that most respondents indicated as supporting an obligation to disclose GIFs were (i) a duty to warn, (ii) respect for autonomy, and (iii) beneficence (Table 3). Although these principles can all be used to defend an obligation to disclose GIFs, they can conflict in the breadth of their implied obligation. For example, relying on a duty-to-warn principle may require disclosing only GIFs that represent significant risk of a serious disease, whereas beneficence may suggest that all potentially useful or relevant GIFs be disclosed. Similarly, basing this obligation on participant autonomy might suggest returning all GIFs and allowing participants to decide for themselves which ones are most important. Again, we hypothesized that professional training and role would be correlated with support for different principles, but this did not seem to be the case.

Support was limited for a number of practical justifications in support of an obligation to disclose, suggesting that our study population found broad philosophical principles to be more persuasive. Only about a third of respondents agreed with the idea that maintaining an institution's professional reputation and avoiding legal liability supported an obligation to disclose GIFs. Interestingly, a relatively large percentage of respondents (19\%) were uncertain about whether a concern for legal liability supported an obligation to disclose GIFs, perhaps because of the lack of existing case law or lack of legal education of respondents. It is possible that views on this rationale for disclosure of GIFs might shift as legal precedent evolves, particularly if the actual risk of liability for nondisclosure increases substantially.$^{18}$

In addition, 34\% of respondents agreed or strongly agreed that an obligation to disclose GIFs rests on a belief that research participants should be treated like clinical patients, with no significant difference between clinicians (40\%), scientists (29\%), and others (33\%), demonstrating that some IRB professionals may see GIFs as existing in a liminal space between 
research and clinical care, whereas many others prefer a firm line between the two. Ascertaining this boundary-or lack thereof-is important: While the American College of Medical Genetics and Genomics (ACMG) has released guidelines for disclosing certain GIFs in the context of clinical genomics, ${ }^{19}$ there have been no equivalent guidelines suggested for the research realm. Instead, many institutions rely on a local advisory board in collaboration with IRBs, which ultimately accept or reject a request to disclose genetic findings. If, as our survey suggests, some IRB professionals believe that research participants should be treated like clinical patients, this may lend credence to concerns about the appropriateness of applying the ACMG guidelines to the research realm. ${ }^{20}$

The other principle that respondents disagreed with most strongly (56\% either disagreed or strongly disagreed) was reciprocity between researchers and participants, or the idea that researchers should disclose GIFs because they owe participants something in exchange for their contribution to the research endeavor. This support for the principle of reciprocity was surprisingly low, given that it is often cited in the literature in support of an obligation to disclose GIFs, ${ }^{16}$ suggesting that these arguments have less traction in the way that IRBs think about the problem of incidental findings. A significant portion of respondents also were unsure about whether an obligation to disclose GIFs is supported by an inherent right of research participants to know their own genetic information, possibly indicating survey respondents' uncertainty about the existence of such a right.

\section{Limitations of an obligation to disclose GIFs}

When asked about the factors or circumstances that could potentially reduce an obligation to disclose GIFs, respondents largely found many of the suggested factors to be unconvincing. In particular-and surprisingly-respondents rejected excuses such as a lack of resources to disclose GIFs or the burden of additional time and effort required to do so, both of which are identified within the literature as significant obstacles to declaring a broad obligation to disclose. ${ }^{18,21}$ Respondents agreed only that inadequate clinical or scientific information reduces an obligation to disclose GIFs. Since both of these factors are based on the current state of science and clinical medicine, it is possible that as the evidence base develops, opinions on whether GIFs should be disclosed may also change.

Survey respondents also rejected arguments based on paternalistic concern for research participants, reaffirming the data indicating that an obligation to disclose GIFs rests at least partly on respect for participant autonomy. Over two-thirds of respondents (70\%) disagreed that an obligation to disclose GIFs is reduced by a worry that participants are not likely to understand genetic risk information, and $67 \%$ of respondents disagreed that the potential psychological impact on participants of learning their genetic risk information is too significant to disclose GIFs.

Interestingly, $73 \%$ of participants either disagreed or strongly disagreed with the idea that the unique role of the clinical investigator, and particularly the lack of clinical responsibilities equivalent to those of practicing physicians, excuses researchers from disclosing GIFs. This suggests that respondents believe clinical researchers should behave similarly to physicians. Most respondents, however, also rejected the idea that research participants should be treated like clinical patients. This inconsistency in beliefs about the difference between research and clinical care again underlines how incidental findings are calling into question the distinction between these two spheres, thereby further complicating efforts to evaluate GIF disclosure policies within clinical research protocols.

\section{Right not to know}

The question of whether research participants have a "right not to know" certain genetic information about themselves continues to be a controversial issue, particularly after the release of the ACMG recommendations. ${ }^{22-24}$ It is clear from our data that while respondents may have strong beliefs about this right in theory, in practice their views are more complicated. Almost all respondents indicated that it is definitely or probably acceptable for a participant to elect not to receive any GIFs, but this strong consensus wavered in the face of specific cases. When considering case $\mathrm{A}$, in which a research team found a highly significant and actionable GIF in the genomic data of a research participant who had elected not to receive any GIFs, a full quarter of respondents said that the research team should either definitely or probably disclose the GIF anyway. In case B, where the original research participant was deceased but had similarly elected not to receive any GIFs, half of respondents thought the research team should either definitely or probably contact the participant's family.

The data supporting these case studies are consistent with the strong consensus that an obligation to disclose GIFs rests on a duty to warn participants who are in significant, imminent danger. It is important, then, for the research ethics community to come to a clear consensus about whether there is truly a strict right for participants not to know any genetic information about themselves, and for this position to be communicated and enforced throughout the informed consent process. Holding an inconsistent position in this area could potentially compromise participant trust in research, as well-meaning researchers disclose information to participants who had previously elected not to receive it. In addition, to ensure that research participants truly understand the meaning and implications of not receiving GIFs, it might be necessary to change the process of consent and have a second discussion about the significance of findings once results are obtained, without disclosing the actual results.

\section{Study limitations}

Our study has several important limitations. First, our response rate was moderate, and nonresponse bias may affect the generalizability of the results. Second, it is possible that subjects were influenced by social acceptability bias. There has been significant discussion about incidental findings, and the field has been moving toward a view that there is an obligation to disclose some set of findings, which could have influenced what 
respondents thought was the desirable answer. Third, our sample primarily comprised nonvoting IRB professionals and was also disproportionately female. While it is possible that nonvoting IRB professionals could hold different views from voting IRB members, or men from women, we found no statistically significant differences between these groups given our sample size. Furthermore, we believe our study population is appropriately representative of the human subjects research ethics community since it was drawn from Public Responsibility in Medicine and Research, the preeminent organization for the research ethics profession. Fourth, we found few differences by professional training or role, although this could have been due to the small size of some of these subgroups. Given these limitations, additional research would be helpful to more confidently assess the views of IRB members, particularly to see how they change over time in this rapidly evolving field.

\section{Conclusion}

The debate about whether to disclose GIFs has been vigorous and continues to evolve, but it has lacked empirical data about the deliberative processes being used to oversee management in the research setting. This study is, to our knowledge, the first exploration of how IRBs are actually grappling with the ethical issues presented by the massive amount of data generated by genomic research. Our research indicates that IRBs are actively engaged with this problem, but like the rest of the field, they have not yet reached clear consensus. The majority of respondents, however, think that return of GIFs is appropriate and have apparent discomfort with arguments that might constrain a duty to return GIFs. This could indicate that IRBs are providing at least some significant portion of the impetus for the trend toward returning incidental findings, although interesting questions remain about the relative contribution of other stakeholders, such as academic bioethicists, researchers, research participants, or funding agencies.

\section{SUPPLEMENTARY MATERIAL}

Supplementary material is linked to the online version of the paper at http://www.nature.com/gim

\section{DISCLOSURE}

The authors declare no conflict of interest. This research was supported in part by the Intramural Research Program of the National Human Genome Research Institute, National Institutes of Health. The opinions expressed here are our own and do not reflect the policies or positions of the National Institutes of Health, the U.S. Public Health Service, or the U.S. Department of Health and Human Services.

\section{REFERENCES}

1. Green ED, Guyer MS; National Human Genome Research Institute. Charting a course for genomic medicine from base pairs to bedside. Nature 2011;470: 204-213.
2. Tabor HK, Berkman BE, Hull SC, Bamshad MJ. Genomics really gets personal: how exome and whole genome sequencing challenge the ethical framework of human genetics research. Am J Med Genet A 2011;155A:2916-2924.

3. Wolf SM, Lawrenz FP, Nelson CA, et al. Managing incidental findings in human subjects research: analysis and recommendations. J Law Med Ethics 2008;36:219-48, 211.

4. Bookman EB, Langehorne AA, Eckfeldt JH, et al.; NHLBI Working Group. Reporting genetic results in research studies: summary and recommendations of an NHLBI working group. Am J Med Genet A 2006;140:1033-1040.

5. Fabsitz RR, McGuire A, Sharp RR, et al. Ethical and practical guidelines for reporting genetic research results to study participants updated guidelines from a National Heart, Lung, and Blood Institute Working Group. Circ Cardiovasc Genet 2010;3:574-580.

6. Beskow LM, Burke W. Offering individual genetic research results: context matters. Sci Trans/ Med 2010;2:38cm20.

7. Miller FG, Mello MM, Joffe $S$. Incidental findings in human subjects research: what do investigators owe research participants? J Law Med Ethics 2008;36:271-9, 211

8. Presidential Commission for the Study of Bioethical Issues. Anticipate and Communicate: Ethical Management of Incidental and Secondary Findings in the Clinical, Research, and Direct-to-Consumer Contexts. Washington, DC, 2013.

9. Williams JK, Daack-Hirsch S, Driessnack M, et al. Researcher and institutional review board chair perspectives on incidental findings in genomic research. Genet Test Mol Biomarkers 2012;16:508-513.

10. Parens $E$, Appelbaum $P$, Chung W. Incidental findings in the era of whole genome sequencing? Hastings Cent Rep 2013;43:16-19.

11. Dressler LG, Smolek S, Ponsaran R, et al.; GRRIP Consortium. IRB perspectives on the return of individual results from genomic research. Genet Med 2012;14:215-222.

12. Smith TW, editor. Standard Definitions Report: Final Dispositions of Case Codes and Outcome Rates for Surveys. 8th edn. AAPOR, 2015.

13. Kaphingst KA, Facio FM, Cheng MR, et al. Effects of informed consent for individual genome sequencing on relevant knowledge. Clin Genet 2012;82:408-415

14. Largent EA, Grady C, Miller FG, Wertheimer A. Money, coercion, and undue inducement: attitudes about payments to research participants. IRB 2012;34:1-8.

15. Ravitsky $\mathrm{V}$, Wilfond $B S$. Disclosing individual genetic results to research participants. Am J Bioeth 2006;6:8-17.

16. Bredenoord AL, Kroes HY, Cuppen E, Parker M, van Delden JJ. Disclosure of individual genetic data to research participants: the debate reconsidered. Trends Genet 2011;27:41-47

17. Eckstein L, Garrett JR, Berkman BE. A framework for analyzing the ethics of disclosing genetic research findings. J Law Med Ethics 2014;42: 190-207.

18. Pike ER, Rothenberg KH, Berkman BE. Finding fault? Exploring legal duties to return incidental findings in genomic research. Georgetown Law J 2014;102:795-843.

19. Green RC, Berg JS, Grody WW, et al.; American College of Medical Genetics and Genomics. ACMG recommendations for reporting of incidental findings in clinical exome and genome sequencing. Genet Med 2013;15:565-574.

20. Jarvik GP, Amendola LM, Berg JS, et al.; eMERGE Act-ROR Committee and CERC Committee; CSER Act-ROR Working Group. Return of genomic results to research participants: the floor, the ceiling, and the choices in between. $A m$ J Hum Genet 2014;94:818-826.

21. Fernandez CV, Kodish E, Weijer C. Informing study participants of research results: an ethical imperative. IRB 2003;25:12-19.

22. American College of Medical Genetics and Genomics. 1 April 1 2014. ACMG updates recommendation on "Opt Out" for genome sequencing return of results. https://www.acmg.net/docs/Release_ ACMGUpdatesRecommendations final.pdf. Accessed 14 July 2015.

23. Burke W, Antommaria AH, Bennett R, et al. Recommendations for returning genomic incidental findings? We need to talk! Genet Med 2013;15:854-859

24. Wolf SM, Annas GJ, Elias S. Point-counterpoint. Patient autonomy and incidental findings in clinical genomics. Science 2013;340:1049-1050. 